\title{
Computing an exact spherical visibility map for meshed polyhedra
}

\author{
Min Liu* Karthik Ramani ${ }^{\dagger}$ \\ Purdue University, West Lafayette, Indiana, USA
}

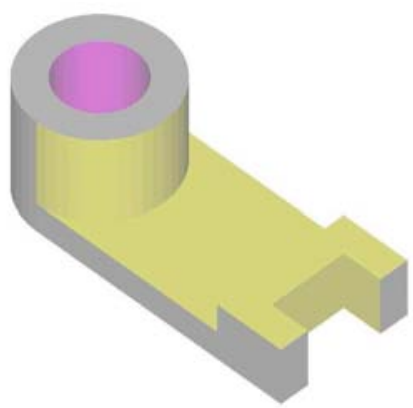

(a)

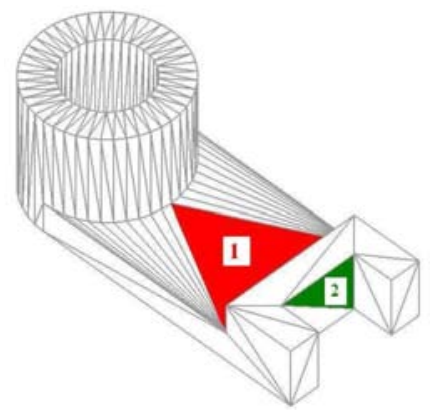

(b)

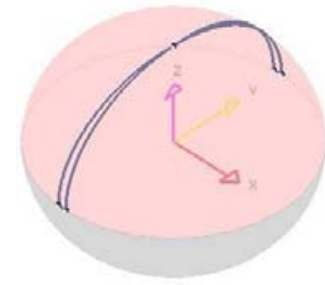

(c)

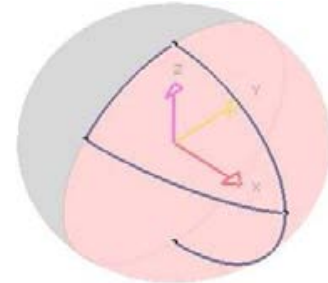

(d)

Figure 1: Spherical visibility maps for triangular facets on a 3D object. (a) A general non-convex 3D object. The two concave regions are colored in yellow and violet respectively. (b) The triangle mesh representation of the object. The two highlighted facets ( $1 \& 2)$ are the exemplar target facets for the visibility illustration. (c) The spherical visibility map for facet 1 (red). It contains a 2-cell bounded by the grey segments. (d) The spherical visibility map for facet 2 (green), which is a 2-cell plus a 1-cell. Each point in the closed visibility map represents a view direction from which the corresponding facet is visible.

\section{Abstract}

This paper considers computation of the exact visibility range (or the spherical visibility map) for a closed polyhedron whose boundary is represented as a triangle mesh. For each facet on the mesh, we calculate the set of view directions from which all the points on the facet can be seen from the exterior. The projection of those visible directions onto the unit sphere forms the visibility map for the facet. We show that the exact visibility map is a spherical arrangement of closed 0-cells, 1-cells, and 2-cells embedded on the surface of the unit sphere. Based on a provable method for calculating the potential occlusion regions of a facet, a vector visibility algorithm is developed for computing the exact solution of the spherical visibility map for a facet. Examples are given to illustrate our algorithm.

Keywords: Spherical visibility map, facet visibility, vector visibility algorithm

\section{Introduction}

Visibility is a fundamental problem that arises in many different contexts in various fields such as computer graphics, computer vision, computational geometry, robotics, CAD/CAM, to name but a few [Durand 2000]. In this paper, we address a specific visibility problem : Given a solid object whose boundary is tessellated as

\footnotetext{
*e-mail: liu66@purdue.edu

†e-mail:ramani@purdue.edu
}

Copyright $\odot 2007$ by the Association for Computing Machinery, Inc.

Permission to make digital or hard copies of part or all of this work for personal or classroom use is granted without fee provided that copies are not made or distributed for commercial advantage and that copies bear this notice and the full citation on the first page. Copyrights for components of this work owned by others than ACM must be honored. Abstracting with credit is permitted. To copy otherwise, to republish, to post on servers, or to redistribute to lists, requires prior specific permission and/or a fee. Request permissions from Permissions Dept, ACM Inc., fax +1 (212) 869-0481 or e-mail permissions@acm.org.

SPM 2007, Beijing, China, June $04-06,2007$.

(c) 2007 ACM 978-1-59593-666-0/07/0006 $\$ 5.00$ a set of triangular facets. For each facet, find the set of view directions (or visible set) from any of which the facet is completely visible.

A point is visible from a view direction if a ray emanating from the point can go into infinity in that direction, without being obstructed by any other entities of the object. The problem is to find the set of directions such that all points in the facet sees the infinity in each of those directions. If we map the set onto a unit sphere, it forms a spherical region called spherical visibility map of the facet.

The study of the above visibility problem is motivated by applications in CAM systems. In spite of the dominance of B-rep approaches, today's more innovative CAM methods are all mesh based, researchers even predict that mesh may well replace BReps as the principal representation of CAM systems [Corney et al. 2005]. Mesh based visibility analysis can be quite useful for process planning in various manufacturing applications. In numerical controlled (NC) machining or in inspection by coordinate measuring machines (CMMs), a feature can be machined or measured only when it can be reached or "seen" by a tool or a probe. The visible set for a feature gives the set of collision free tool access directions [Scott et al. 2003; Kweon and Medeiros 1998; Balasubramanian et al. 2000]. In mold design, each movable mold piece associates a parting direction from which the mold should be opened without interaction with other pieces and the object in the mold itself. Therefore, the parting direction for a mold piece has to be one of the visible directions of the corresponding surface region formed by a particular mold piece [Ravi and Srinivasan 1990; Priyadarshi and Gupta 2004].

Woo [1994] first developed the concept of the spherical visibility map and linked the visibility problem to various manufacturing processes. This visibility map defines the set of view directions free of self occlusion instead of the global occlusion [Chen et al. 1993]. The same idea has been explored by Kim et al. [1995] to cover the Bézier surfaces and Elber and Cohen [1995] to cover the freeform surfaces. 
Raster visibility methods for computing the visible set for 3D object were targeting the building of a visibility matrix based on the discretized approximation of the solution space [Tarbox and Gottschlich 1996; Balasubramanian et al. 2000; Spitz and Requicha 2000; Khardekar et al. 2006]. The elements of the visibility matrix encode the visibility of discrete object surface entities from each sampled point in a quantized viewing sphere. This changes the problem to it dual - the classical hidden surface removal problem. The drawback of this sampling-and-testing approach is that no matter how good the sampling scheme is in the solution space, it is still possible to falsely report some visible entities as invisible.

A few vector visibility methods have treated the solution space as continuous [Nirenstein et al. 2002; Mora et al. 2005; Bittner et al. 2005; Keeler et al. 2007]. Dhaliwal et al. [2003] presented an algorithm for computing the global accessability for polyhedra objects. But their method tessellated the viewing sphere into spherical triangles and ignored the degenerate cases of a visibility map which happens frequently for mechanical parts. For examples shown in Figure. 1(d), 5(a) and 5(b), their method ends up with incomplete or false solutions. In a latest work on the hidden surface removal, the spherical visibility maps are generated in a different context [Keeler et al. 2007] with a similar spherical representation and solution that we have approached. This work, coincidentally and fortunately, bridges the two dual problems: the hidden surface removal under perspective projection and the spherical visibility map under orthographic projection into the same solution space and the same representation schema.

Two somewhat related problems in computational geometry are the computation of the aspect graph and the visibility complex. The former partitions viewpoint space according to the qualitative aspect of view [Eggert et al. 1992] while the later deals with the creation of a data structure that encodes all visibility relations between objects in the collection [Pocchiola and Vegter 1996].

We developed a method to find the exact boundaries of the spherical visibility map. Figure 1 shows an example of the input and output of our method. We differentiate our work from the above cited and others by developing a theoretically exact solution and the associated representation of the visible set embedded on the unit sphere.

\section{The Spherical Visibility Map}

\subsection{Preliminaries}

We first briefly review some terms that will be used in sequence and a few elements from spherical geometry.

The viewing sphere (or unit sphere), $S^{2}$, represents the manifold of all unit vectors starting from origin. A spherical point is a point on $S^{2}$ which corresponds to a nontrivial view direction. Two spherical points are antipodal to each other if the two corresponding view directions are opposite. A spherical circle is an oriented great circle on $S^{2}$. The orientation of the great circle is that of a counterclockwise walk along the circle as seen from the positive half space of the oriented plane containing the circle. A spherical segment is a continuous subset of a spherical circle which is bounded by two spherical points. A spherical polygon is a connected region on $S^{2}$ which is bounded by one outer cycle of spherical segments and an arbitrary number of inner cycles of spherical segments, with the interior to the left of each segment. A spherical polygon is convex if any spherical segment bounded by two spherical points of the spherical polygon is contained inside the spherical polygon.
We give three types of spherical arrangement of point set on $S^{2}$ specific names, which are: 0 -cell, 1-cell, and 2-cell. A 0 -cell is an isolated spherical point. A 1-cell is the set of connected spherical edges. A 2-cell is a spherical polygon with or without holes.

The local visibility map (or LVMap) of a facet $f$ is the set of points on $S^{2}$ that corresponds to all directions $\vec{v}$, satisfying $\vec{v} \cdot \vec{n}_{f} \geqslant 0$. LVMap of a facet is a closed hemisphere on $S^{2}$, with the facet normal as the pole, and the spherical circle defined by the intersection of the carrying plane $\mathscr{P}(f)$ and $S^{2}$ as the boundary. The spherical visibility map (or SVM) of facet $f$ is the set of points on $S^{2}$ that correspond to the occlusion free directions of $f$.

For facet $f$, it divides the three-space into two half spaces separated by $\mathscr{P}(f)$. The closed half space on the side of $f$ 's normal points is called positive half space of $f$, or $H^{+}(f)$, the interior of $H^{+}(f)$ is called the up space of $f$. The other closed half-space is called negative half space of $f$, or $H^{-}(f)$, with the corresponding interior as down space of $f$.

Given mesh $M$, we call facet $f$ on $M$ a hull facet, if and only if, $M \subset H^{-}(f)$; otherwise, $f$ is a non-hull facet. The connected set of non-hull facets on $M$ is called a concave region.

A pair of facets $f$ and $f^{\prime}$ face each other if the following conditions are satisfied: (1) $f$ and $f^{\prime}$ are not coplanar; (2) $f$ lies fully or partially in $H^{+}\left(f^{\prime}\right)$, and (3) $f^{\prime}$ lies fully or partially in $H^{+}(f)$.

Property 1: The SVM of a hull facet is a bounded hemisphere which is equal to its local visibility map.

Property 2: A ray emanating from a non-hull facet will either intersect a facet in the same concave region or go to infinity. [Chen et al. 1993; Dhaliwal et al. 2003].

Property 3: A pair of non-hull facets can obstruct their mutual visibility only if they are facing each other.

Property 4: The SVM of a non-hull facet is a subset of its local visibility map, and it is not connected in general.

\subsection{Occlusion region for a pair of facing facets}

In this section, we explore the different facing conditions and the corresponding properties of the introduced occlusion region. Given facet $f$, its facing facet $f^{\prime}$ may have one of the following space relationships to $f$. We name them as different $f^{\prime}$-to- $f$ configurations.

Above: $f^{\prime}$ lies entirely in the up space of $f$ (Figure2(a)).

Cross: $f^{\prime}$ lies partially in the up space of $f$ and partially in the down space of $f$ (Figure2(b)). The line segment of $f^{\prime}$ which lies on the the plane $\mathscr{P}(f)$ is called the crossing edge.

Vertex-on: $f^{\prime}$ lies in the positive half space of $f$ and $f^{\prime}$ has only a vertex touching the plane $\mathscr{P}(f)$ (Figure2(c)). This vertex is called a touching vertex.

Edge-on: $f^{\prime}$ lies in the positive half space of $f$ and $f^{\prime}$ has an edge on the plane $\mathscr{P}(f)$ (Figure2(d)). This edge is called a touching edge.

If $f^{\prime}$-to- $f$ is a 'Cross' configuration, $f^{\prime}$ can be clipped into two facets $f_{1}^{\prime}$ and $f_{2}^{\prime}$ along the crossing edge. $f_{1}^{\prime}$ is in the positive half space of $f$ while $f_{2}^{\prime}$ belongs to the negative half space of $f$ (see Figure4(c)). Obviously only $f_{1}^{\prime}$ will be able to block $f^{\prime}$ 's visibility and it forms an 'Edge-on' configuration to the facet $f$. In the following occlusion region calculation, $f^{\prime}$ should be replaced by $f_{1}^{\prime}$ if $f^{\prime}$-to- $f$ is a 'Cross' configuration. 


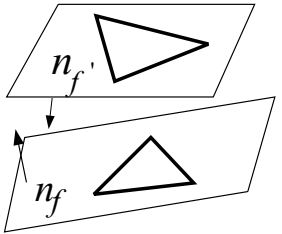

(a) Above

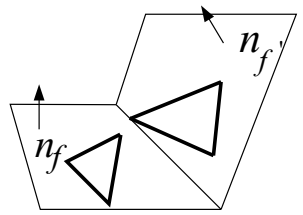

(c) Vertex-on

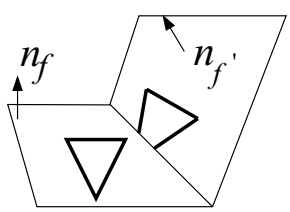

(b) Cross

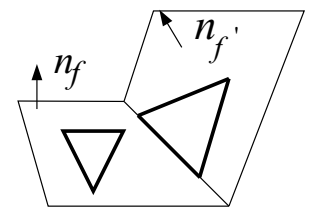

(d) Edge-on
Figure 2: Different cases of $f^{\prime}$-to- $f$ configurations

Theorem 2.1 The occlusion region OMap $\left(f, f^{\prime}\right)$ of a convex polygonal facet $f$ due to another convex polygonal facet $f^{\prime}$ is the spherical convex hull ( $\mathrm{SCH}$ ) of all the spherical points describing the set of extremal stabbing lines.

Proof: What needs to be proved is that OMap $\left(f, f^{\prime}\right)$ is convex. Then it should follow that it is the spherical convex hull of the spherical points describing the extremal stabbing lines.

Any vector from a point in $f$ to another point in $f^{\prime}$ describes a spherical point on $S^{2}$. The union of all such spherical points forms the OMap $\left(f, f^{\prime}\right)$. Take two directions $v_{1}$ and $v_{2}$ in OMap $\left(f, f^{\prime}\right)$. It means that there are two points $M_{1}$ and $M_{2}$ on $f$ such that the rays $\left(M_{1}, v_{1}\right)$ and $\left(M_{2}, v_{2}\right)$ are blocked by $f^{\prime}$, which they intersect at points $P_{1}$, and $P_{2}$. To prove OMap $\left(f, f^{\prime}\right)$ is convex, we just need to show that any direction $v$ "in between $v_{1}$ and $v_{2}$ " (the spherical points in the spherical segment bounded by $v_{1}$ and $v_{2}$ ), there is a point $M$ in line segment $\overline{M_{1} M_{2}}$ such that the ray $(M, v)$ intersect $f^{\prime}$ at $\mathrm{P}$ in the line segment $\overline{P_{1} P_{2}}$. Thus by convexity of $f$ and $f^{\prime}, M$ is in $f$ and $P$ is in $f^{\prime}$, we can conclude that $v$ is in OMap $\left(f, f^{\prime}\right)$, and OMap $\left(f, f^{\prime}\right)$ is convex.

For the line segment $\overline{M_{1} M_{2}}$ in $f$, and the line segment $\overline{P_{1} P_{2}}$ in $f^{\prime}$, we show that the OMap $\left(\overline{M_{1} M_{2}}, \overline{P_{1} P_{2}}\right)$ is the spherical convex hull of the four points describing the four extremal stabbing lines.

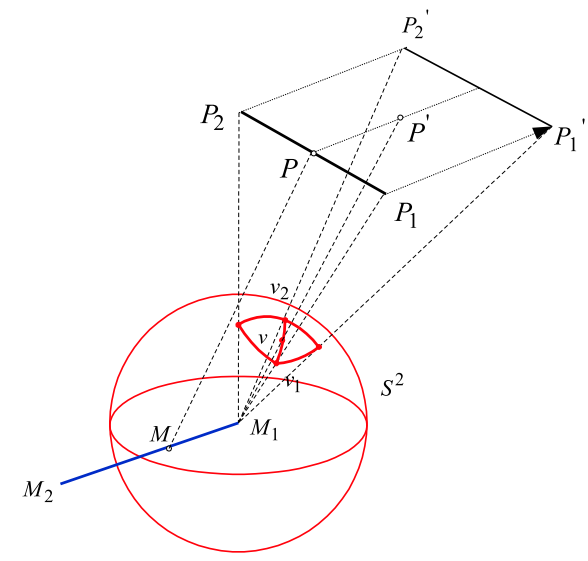

Figure 3: Occlusion region of a line segment due to another line segment
If we start from $M_{1}$ and initialize the origin of $S^{2}$ at $M_{1}$, OMap $\left(M_{1}, \overline{P_{1} P_{2}}\right)$ is the spherical projection of $\overline{P_{1} P_{2}}$ onto $S^{2}$. Sweep the center of the unit sphere along $\overline{M_{1} M_{2}}$ to $M_{2}$, and project $\overline{P_{1} P_{2}}$ continuously onto $S^{2}$ results in a series of spherical segments. The union of these spherical segments will be equal to the spherical projections when we fix the unit sphere at $M_{1}$, and sweep the line segment $\overline{P_{1} P_{2}}$ along the direction $\overrightarrow{M_{2} M_{1}}$ by the length of $\left|\overline{M_{2} M_{1}}\right|$ (See Figure 3 for illustration).

If $\overline{M_{2} M_{1}}$ is not parallel to $\overline{P_{1} P_{2}}$, sweeping of $\overline{P_{1} P_{2}}$ forms a parallelogram $\mathbb{P}$ (see $P_{1} P_{1}^{\prime} P_{2}^{\prime} P_{2}$ in Figure 3 ). OMap $\left(\overline{M_{1} M_{2}}, \overline{P_{1} P_{2}}\right.$ ) will be equal to OMap $\left(M_{1}, \mathbb{P}\right)$, which is the spherical projection of the $\mathbb{P}$ onto the $S^{2}$ centered at $M_{1}$. OMap $\left(M_{1}, \mathbb{P}\right)$ is a convex polygon bounded by four points describing the four vectors: $\overrightarrow{M_{1} P_{1}}, \overrightarrow{M_{1} P_{2}}$, $\overrightarrow{M_{1} P_{1}^{\prime}}$ and $\overrightarrow{M_{1} P_{2}^{\prime}}$. Among them $\overrightarrow{M_{1} P_{1}^{\prime}}=\overrightarrow{M_{2} P_{1}}$ and $\overrightarrow{M_{1} P_{2}{ }^{\prime}}=\overrightarrow{M_{2} P_{2}}$, thus the four stabbing line defines the four bounding points of OMap $\left(M_{1}, \mathbb{P}\right)=$ OMap $\left(\overline{M_{1} M_{2}}, \overline{P_{1} P_{2}}\right)$.

Due to the convexity of this OMap, the spherical segment $\overline{v_{1} v_{2}}\left(v_{1}\right.$ describes $\overrightarrow{M_{1} P_{1}}$, and $v_{2}$ describes $\overrightarrow{M_{2} P_{2}}$ ) is also contained in OMap $\left(\overline{M_{1} M_{2}}, \overline{P_{1} P_{2}}\right)$. For any point $\mathrm{v}$ in $\overline{v_{1} v_{2}}$, the ray $\left(M_{1}, v\right)$ intersects $\mathbb{P}$ at point $P^{\prime}$ in $\mathbb{P}$. We can construct another parallelogram by sweeping $\overline{M_{1} P}$ along direction $\overrightarrow{M_{1} M_{2}}$ till $P^{\prime}$ intersects $\overline{P_{1} P_{2}}$ at point $P$, and $M_{1}$ goes to $M$. Since $\overline{M P} \| \overline{M_{1} P^{\prime}}$, the ray $(M, v)$ is blocked by the point $\mathrm{P}$.

If $\overline{M_{1} M_{2}} \| \overline{P_{1} P_{2}}$, the above sweeping forms a new line segment, and the above arguments still hold true for this trivial case. $\square$

In general, the bounding segments of an occlusion region represent view directions where the corresponding rays just touch the boundaries of $f^{\prime}$ and still goes to infinity; they are not part of the occlusion region. But, there are some special cases which need to be considered. Given facet $f$, and one of its facing facet, $f^{\prime}$. If $f^{\prime}$-to- $f$ is 'Cross', 'Edge-on' or 'Vertex-on' configuration, OMap $\left(f, f^{\prime}\right)$ has intersected spherical segments with the bounding circle of $f$ 's LVMap. We call this spherical segment on the bounding circle a crucial arc (e.g. the solid red arc on the Figure4(c)). For the boundary conditions of $\operatorname{OMap}\left(f, f^{\prime}\right)$ on the crucial arcs, we have the following proposition:

Proposition 2.2 If $f^{\prime}$-to- $f$ is 'Cross' configuration, $f$ is occluded from the interior of the crucial arc. The crucial arc, which includes none of its bounding points, belongs to the OMap $\left(f, f^{\prime}\right)$.

If $f^{\prime}$-to- $f$ is 'Edge-on' configuration, the open crucial arc belongs to the OMap $\left(f, f^{\prime}\right)$ if the touching edge has an internal angle greater than the angle between the two facet normals $n_{f^{\prime}}$ and $n_{f}$.

Proof: The crucial arc denotes the range of ray directions that emanate from facet $\mathrm{f}$, parallel to the carrying plane of $f$, and just crosses the touching edge or touching vertex of $f^{\prime}$ (for 'Cross' type configuration, it's the touching edge of $f_{1}^{\prime}$ ).

For 'Cross' type configurations, these rays will be blocked by $f^{\prime}$ itself because they'll find no way to go inwards to the material side of the facet $f^{\prime}$ (see Figure4(c)). The only two exceptions happen at the two bounding points of the crucial arc, those two rays hit the bounding vertices of the touching edge; they won't be blocked by $f^{\prime}$ itself.

For the 'Edge-on' type configurations, the material exists in between $f^{\prime}$ and its neighboring facet, which shares the touching edge of $f^{\prime}$. If the internal angle (the angle measured from the material side) between these two neighboring facets, $\alpha_{1}$ is greater than the angle between $n_{f^{\prime}}$ and $n_{f}, \alpha_{2}$ (Figure4(a)), it means that $f^{\prime}$ 's neighboring facet lies blow the carrying plane of $(f)$ so that the rays can not go forward into the material between $f^{\prime}$ and its neighboring facets. Two exceptions are on the bounding points of the crucial 


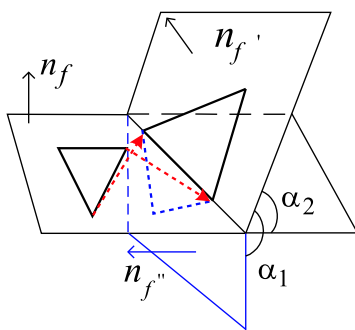

(a) 'Edge-on': $\alpha_{1}>\alpha_{2}$

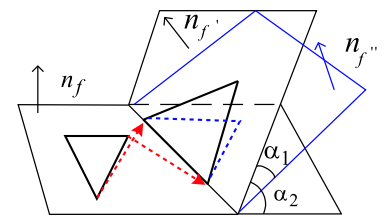

(b) 'Edge-on': $\alpha_{1}<\alpha_{2}$
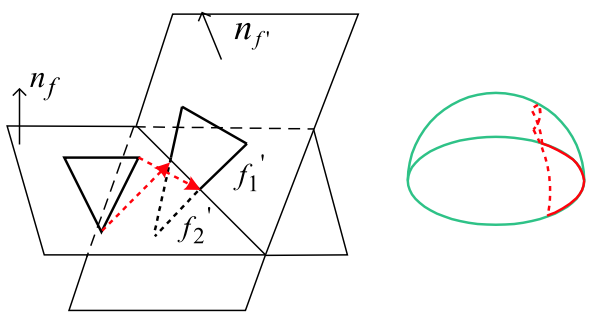

(c) 'Cross' type configuration. The red solid arc represents the crucial arc, which is part of $\operatorname{OMap}\left(f, f^{\prime}\right)$.

Figure 4: Illustration of the boundary conditions of the crucial arcs on $\operatorname{OMap}\left(f, f^{\prime}\right)$.

arc as those in above for the 'Cross' configurations. Otherwise, if $\alpha_{1} \leqslant \alpha_{2}$ (see Figure4(b)), $f^{\prime}$ and its neighbor can not block the range of rays on the carrying plane of $f$. Therefore, the crucial arc is not part of $\operatorname{OMap}\left(f, f^{\prime}\right)$. $\square$

If $f^{\prime}$-to- $f$ is 'Vertex-on' configuration, the crucial arc on OMap ( $f$, $f^{\prime}$ ) represent the rays that emanate from $f$ and hit the touching vertex of $f^{\prime}$. Those ray directions are not part of the OMap $\left(f, f^{\prime}\right)$.

\subsection{SVM for a non-hull facet}

For each facet $f^{\prime}$ that faces $f$ and presents in the same concave region as $f, f^{\prime}$ will introduce an occlusion region OMap in $f^{\prime}$ 's SVM. If $f$ 's SVM is initialized as its LVMap, given the presence of $f^{\prime}$, the occlusion region OMap $\left(f, f^{\prime}\right)$ should be subtracted from the SVM of $f$.

$$
S V M(f) \leftarrow S V M(f)-O M a p\left(f, f^{\prime}\right)
$$

Iterating the above subtraction for every eligible facing facet $f^{\prime}$ will finally result in the exact SVM of a non-hull facet $f$.

Considering the spherical arrangement of the final SVM, which is constructed by the above iterative process, we have the following proposition.

Proposition 2.3 The SVM of a non-hull facet is a spherical arrangement which contains only 0-cells, 1-cells, or 2-cells. All of these cells are closed in the hemispherical LVMap of the facet.

Proof: First of all, the SVM of a non-hull facet is a subset of its LVMap; therefore, every possible spherical arrangement of SVM, no matter what type of cells it contains, is always enclosed by the hemispherical LVMap of the facet. The boundaries of the SVM represent those of ray directions emanating from the non-hull facet, touching the boundaries of some facing facets in the same concave region, and finally reaching the infinity outside of the object. Those ray directions correspond to the visible view directions of the facet.
The exact SVM of a non-hull facet can be considered as the result of a successive removal of some open 2-cell OMaps (convex polygons) from a closed hemispherical LVMap. In the cases of the 'Cross' and 'Edge-On' type of configurations, the open 2-cell may contain partial of the 2-cell boundaries, which is the crucial arc. Crucial arcs happen only on the boundary of the LVMap. The successive subtractions can result in only three types of cells: 0-cell, 1cell, or 2-cell. 0-cell results when a spherical point is present on the boundaries of some OMaps but not inside of any OMap. 1-cell happens when some of the 2-cell OMaps have overlapping boundaries arc, and the overlapping arc is not contained by any other OMaps. 2 -cell is the common result when subtracting a 2-cell from another. Figure 5 gives examples of a 0-cell, 1-cell and 2-cell type of SVMs.
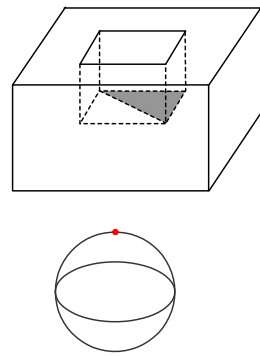

(a) 0-cell SVM
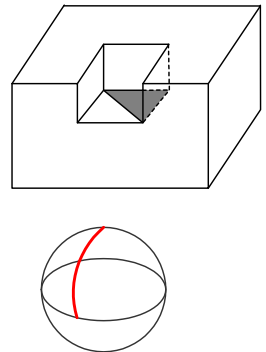

(b) 1-cell SVM
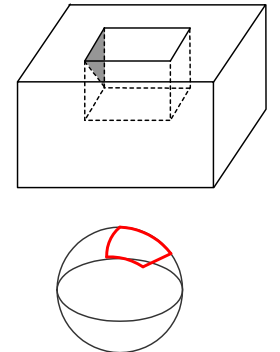

(c) 2-cell SVM
Figure 5: Spherical arrangement of SVMs

\section{Algorithms for computing exact SVM}

The general idea for calculating SVM for a non-hull facet $f$ has been introduced when we discussed the spherical arrangement of SVM. That is, (1)Map all the possible rays emanating from $f$ onto the unit sphere. This gives the initialized SVM of $f$ : a hemispherical 2-cell. (2) For each of $f$ 's facing facets $f^{\prime}$, calculate the occlusion region: OMap of $f$ due to $f^{\prime}$. (3) Calculate the union of all the OMaps and subtract it from SVM of $f$.

Since the input is a triangle mesh, a preprocessing step is performed to group the neighboring facet in each concave region into convex polygons if there is one. We calculate the occlusion region based on the facing convex polygons.

The detailed algorithms for computing SVM for a facet are listed below with pseudo-codes. The Algorithm VISIBILITY-MAP takes a non-hull facet and its associated concave region as input, and outputs the VMap for the particular facet. See Algorithm 1.

For obtaining the OMap $\left(f, f^{\prime}\right)$, there is a need for calculating a spherical convex hull. Method exists by central projection of the spherical points onto a suitable tangent plane of the unit sphere, thus transferring the problem to the standard 2D convex hull calculation ([Khardekar et al. 2006]). How ever, selecting a suitable tangent plane itself is a non-trivial problem. Qian and Harding [2003] developed a $O\left(n^{2}\right)$ rolling cutting plane method for solving the spherical convex hull. Here we propose a direct extension of Graham's scan algorithm for planar convex hull calculation to the spherical space for computing the spherical convex hull, with time complexity $O(n \log (n)$. See the details in Algorithm 2. 

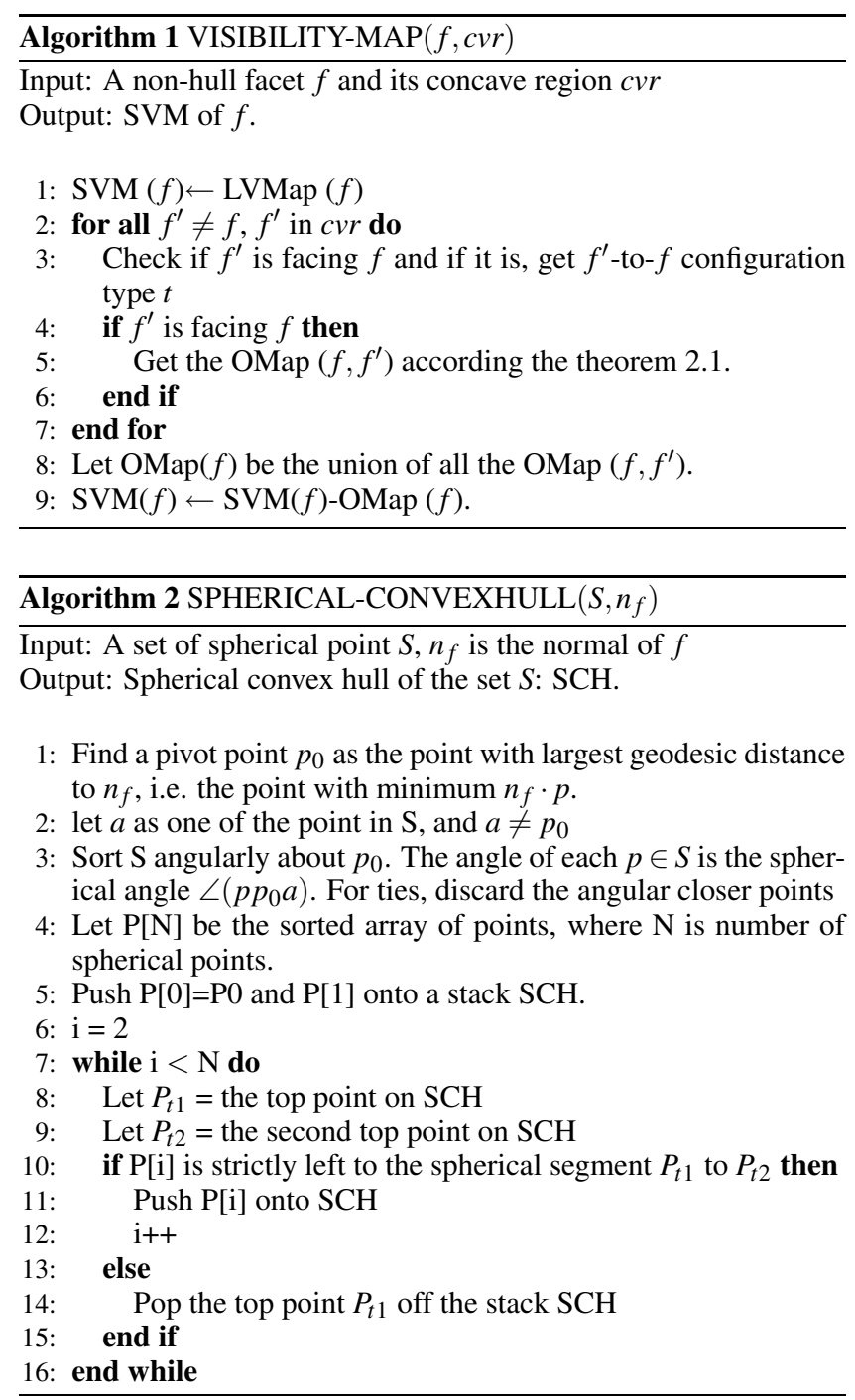

\section{Implementation and discussion}

All the above-described algorithms have been implemented on Windows XP using Visual C++. We interfaced with the two open source algorithm implementations. For determining whether a facet is hull facet or non-hull facet, we used the Quickhull algorithm implementation from the online resource [Qhull ]. For the Boolean operations of polygons embedded on the unit sphere (step 8 in Algorithm 1), we interfaced with the Nefpolydedra data structure and the related algorithms implemented in Computational Geometry Algorithms Library [CGAL ]. Cartesian kernel with the Gmpq (arbitrary precision rational number) number type, which uses the exact arithmetic for numerical operations is utilized to ensure the robustness of the geometric calculations.

In the current implementation, the major running time is spent on the spherical Boolean operations. The current CGAL libraries support only boolean operations of two polygons at a step. Each step takes time $O((n+k) \log n)$, where $n$ is the number of input spherical segments and $k$ is the number of output spherical segments in each step. Since all the OMaps are co-hemispherical, the union of the OMaps could actually be implemented by an adaption of the conventional plane sweeping algorithm [de Berg et al. 2000] using a half-circle, with time complexity of $O\left(\left(n^{\prime}+k^{\prime}\right) \log n^{\prime}\right)$, where $n^{\prime}$

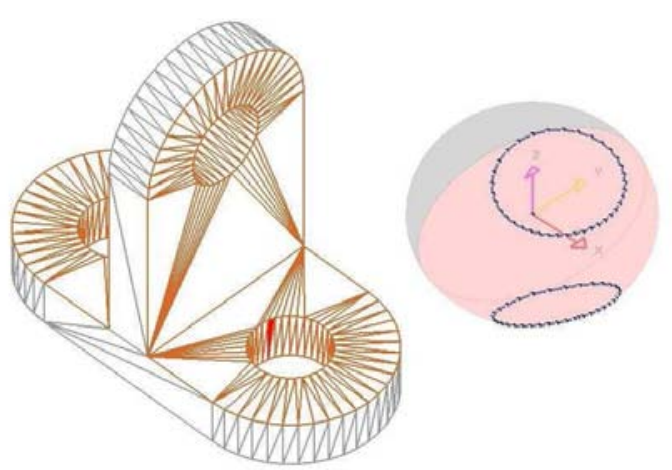

(a) Bracket

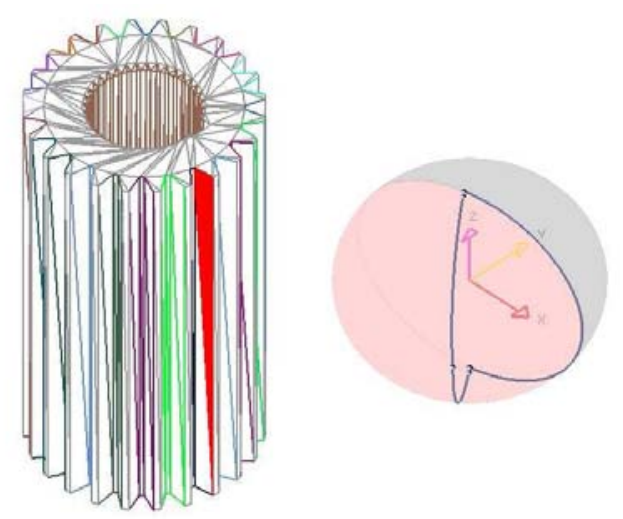

(b) Gear part

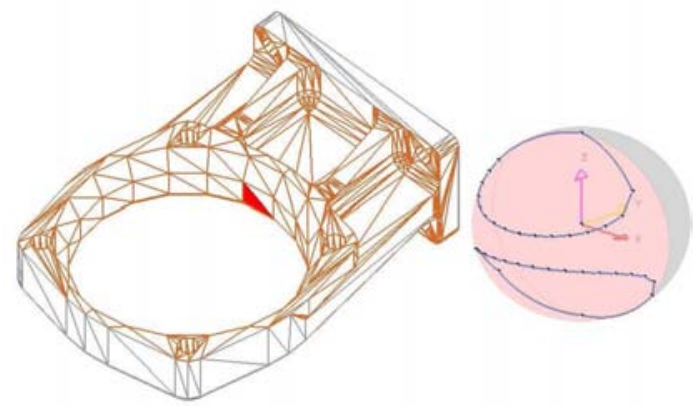

(c) Casting part

Figure 6: Testing examples and their SVMs

Table 1: Implementation results.

\begin{tabular}{llll}
\hline Model & $\begin{array}{l}\text { Bracket } \\
\text { Figure 6(a) }\end{array}$ & $\begin{array}{l}\text { Gear } \\
\text { Figure 6(b) }\end{array}$ & $\begin{array}{l}\text { Casting } \\
\text { Figure 6(c) }\end{array}$ \\
\hline \# Facets & 736 & 820 & 1652 \\
\# Concave Regions (CR) & 1 & 25 & 1 \\
\# Facets in same CR & 582 & 6 & 1445 \\
\# Facing polygons & 36 & 2 & 152 \\
\hline \# Spherical Pnts in SVM & 78 & 9 & 80 \\
\# Execution time (secs) & 3 & $<0.01$ & 55 \\
\hline
\end{tabular}


is the total number of the spherical segments in the input spherical polygons, and $k^{\prime}$ is the number of intersections among the spherical segments and can be at most $n^{\prime 2}$. In total this leads to a worst case time complexity of $O\left(n^{\prime 2} \log n^{\prime}\right)$. But in general, $k^{\prime}$ is largely smaller than $n^{\prime 2}$.

Figure 6 shows some of our testing results (on Pentium IV $1.20 \mathrm{GHz}$ processor, with 512M RAM) for three different CAD objects. The facets in the same concave region are rendered with the same color. The targeting facets are colored in red, with their corresponding SVMs shown to the right (Pink regions show the LVMaps for the highlighted facet, SVM for each facet is the closed 2-cells bounded by the grey spherical segments. Table 1 gives the correspond statistics on the input and output data of the testing examples). Because of the above analyzed time complexity and the overhead of the exact arithmetic used in CGAL library, it is a little slower than expected. This makes the current implementation practical only for relatively simple objects. The testing result shows that our SVM computing method is robust and precise. For the efficiency issue, we leave the implementation of the plane sweeping algorithm on sphere with lower time complexity for our future work.

\section{Conclusions}

In this paper, we have presented an exact algorithm for determining the set of view directions from which a triangular facet is visible. The visible set is proven to be an arrangement of different topological cells embedded on the unit sphere. A spherical visibility map is proposed to represent the visible set of a facet. A provable sound method has been developed for computing the occlusion region for a facet due to the presence of another convex polygonal facet. We differentiated four facing conditions and derived exact descriptions of the boundaries for an occlusion region. Based on that, a vector visibility method has been developed to compute the spherical visibility map for meshed polyhedra.

\section{References}

BAlasubramanian, M., LAXMiPRASAD, P., SARMA, S., AND SHAIKH, Z. 2000. Generating 5-aixs nc roughing paths directly from a tessellated representation. Comput. Aided Des. 32, 4, 261-277.

BitTner, J., Wonka, P., AND Wimmer., M. 2005. Fast exact from-region visibility in urban scenes. 223-230. In Proc. of Eurographics Symposium on Rendering 2005.

CGAL. Cgal reference manual. 3.1 ed, http://www . cgal .org.

Chen, L.-L., Chou, S.-Y., And Woo, T. C. 1993. Separating and intersecting spherical polygons: computing machinability on three-, four-, and five-axis numerically controlled machines. ACM Trans. Graph. 12, 4, 305-326.

Corney, J., Hayes, C., Sundararjan, V., And Wright, P. 2005. The cad/cam interface: A 25-year retrospective. J. of Computing and Information Science in Engineering 5, 3, 188197.

De Berg, M., VAn Kreveld, M., Overmars, M., And SCHWARZKOPF, O. 2000. Computational Geometry: Algorithms and Applications. 2nd Edition, Springer-Verlag 2000.

Dhaliwal, S., Gupta, S. K., And Priyadarshi, J. H. A. 2003. Algorithms for computing global accessiblity cones. J. of
Computing and Information Science In Engineering 3, 3, 200209.

DURAND, F. 2000. A multidisciplinary survey of visibility, ACM SIGGRAPH course notes.

Eggert, D. W., Bowyer, K. W., AND Dyer, C. R. 1992. Aspect graphs: State-of-the-art and applications in digital photogrammetry. In Proc. 17th Cong. Int. Soc. Photogrammetry Remote Sensing, 633-645.

Elber, G., And Cohen, E. 1995. Arbitrarily precise computation of gauss maps and visibility sets for freeform surfaces. In SMA '95: Proc. of the third ACM symposium on Solid modeling and applications, ACM Press, New York, NY, USA, 271-279.

KeEler, T., Fedorkiw, J., And GHALI, S. 2007. The spherical visibility map. Comput. Aided Des. 39, 1, 17-26.

Khardekar, R., Burton, G., And McMains, S. 2006. Finding feasible mold parting directions using graphics hardware. Comput. Aided Des. 38, 9, 741-749.

Kim, D.-S., Papalambros, P. Y., And Woo, T. C. 1995. Tangent, normal, and visibility cones on Bézier surfaces. Comput. Aided Geometric Des. 12, 3, 305-320.

Kweon, S., And Medeiros, D. J. 1998. Part orientations for $\mathrm{cmm}$ inspection using dimensioned visibility maps. Comput. Aided Des. 30, 9, 741-749.

Mora, F., Aveneau, L., And Mériaux, M. 2005. Coherent and exact polygon-to-polygon visibility. WSCG'2005, Plzen, République Tchèque.

Nirenstein, S., Blake, E., And Gain, J. 2002. Exact fromregion visibility culling. In EGRW'02: Proc. of the 13th Eurographics workshop on Rendering, Eurographics Association, Aire-la-Ville, Switzerland, Switzerland, 191-202.

Pocchiola, M., And Vegter, G. 1996. The visibility complex. Int. J. of Computational Geometry and Applications, 6, 275-308.

PriYAdARShi, A. K., AND GUPTA, S. K. 2004. Geometric algorithms for automated design of multi-piece permanent molds. Comput. Aided Des. 36, 3, 241-260.

QHULL. Qhull code for convex hull, http://www.qhull.org/.

QIAN, X., AND HARDING, K. G. 2003. Computational approach for optimal sensor setup. Optical Engineering 42, 5, 1238-48.

RaVi, B., AND SRInivasan, M. N. 1990. Decision criteria for computer-aided parting surface design. Comput. Aided Des. 22, $1,11-18$.

Scott, W. R., Roth, G., And Rivest, J.-F. 2003. View planning for automated three-dimensional object reconstruction and inspection. ACM Computing Surveys 35, 1, 64-96.

Spitz, S. N., AND Requicha, A. A. G. 2000. Accessibility analysis using computer graphics hardware. IEEE Trans. on Visualization and Computer Graphics 6, 3, 208-219.

Tarabanis, K., Tsai, R. Y., And Kaul, A. 1996. Computing occlusion-free viewpoints. IEEE Trans. on Pattern Analysis and Machine Intelligence 18, 3, 279-292.

Tarbox, G., And Gottschlich, S. 1996. Planning for complete sensor coverage in inspection. Computer Vision and Image Understanding 61, 1, 84-111.

Woo, T. C. 1994. Visibility maps and spherical algorithms. Comput. Aided Des. 26, 1, 6-16. 